\title{
Hierarchical Classification of Web Documents by Stratified Discriminant Analysis
}

\author{
Juan Carlos Gomez and Marie-Francine Moens \\ Katholieke Universiteit Leuven \\ Department of Computer Science, Celestijnenlaan 200A, B-3001 Heverlee, Belgium \\ juancarlos.gomez@cs.kuleuven.be, sien.moens@cs.kuleuven. be
}

\begin{abstract}
In this work we present and evaluate a methodology to classify web documents into a predefined hierarchy using the textual content of the documents. Hierarchical classification using taxonomies with thousands of categories is a hard task due to the problem of scarcity of training data. Hierarchical classification is one of the rare situations where, despite the large amount of available data, as more documents become available, more classes are also added to the hierarchy. This leads to a lack of training data for most of the categories, which produces poor individual classification models and tends to bias the classification to dense categories. Here we propose a novel feature extraction technique called Stratified Discriminant Analysis (sDA) that reduces the dimensions of the text content features of the web documents along the different levels of the hierarchy. The sDA model is intended to reduce the effects of scarcity of data by better grouping and to identify the categories with few training examples leading to more robust classification models for those categories. The results of classifying web pages from the Kids\&Teens branch of the DMOZ directory show that our model extracts features that are well suited for category grouping of web pages and representation of categories with few training examples.
\end{abstract}

Keywords: hierarchical classification, text content, web mining, discriminant analysis

\section{Introdution}

In the current information era, classification of documents and digital content plays a very important role in many information management and retrieval tasks. On the web, the number of documents created daily is huge and there is a demand for organizing them in some manner. In this sense, one valuable approach for the management of information is to have the web documents organized by means of hierarchies of conceptual categories, since the use of such hierarchies simplifies the abstraction and facilitates the access to information [8]. In this direction, most of the famous browsing directories (e.g. ODP, Yahoo!) give access to web documents that are manually classified inside a hierarchy of categories. Nevertheless, as the Web changes and grows exponentially, the existing number 
of non-classified web documents largely exceeds the number of these manually labeled inside the hierarchies of the systems. Then it is easy to imagine building automatic classifiers in order to reduce the amount of manual labor on such task. These automatic classifiers, could help to maintain, update and expand web directories [8]; to build customized and relevant views of such directories depending on the necessities of the user, improving the accessibility of content; to improve the quality of content search by returning clustered results [7][9]; and to build efficient crawlers on search engines, among other tasks [25].

In hierarchical classification (HC), categories are arranged as nodes in a graph which represents a hierarchy, usually in the form of a tree. The problem of HC is then defined as: given a hierarchy of categories $\mathfrak{H}$, to automatically classify web documents inside $\mathfrak{H}$ by assigning to the documents a set of categories in a topdown manner, from most general to most specific topics. In general, it is assumed that when a document is categorized with a certain set of node categories from the hierarchy, the document belongs as well to all the node categories along the path connecting the assigned nodes to the root of the graph.

$\mathrm{HC}$ has been studied by several researchers in the past years, developing interesting methods ([1][3][5][6][7][8][19][21][29]). In general there are two approaches to solve the problem [26][29]: the top-down approach (indeed the HC approach), which exploits the hierarchy by dividing the problem into smaller ones; and the flat approach, which directly categorize the documents, ignoring the hierarchy. The HC approach simplifies the problem, but it tends to propagate errors made at higher levels of the hierarchy. On the other hand, the flat approach does not propagate errors but tends to be expensive in time for large hierarchies with thousands of categories. In both cases, there is the problem of scarcity, where for large web directories the number of documents could be huge but as well is the number of categories [15][19][23]. Then, most of the categories contain a small number of labeled documents, offering little information for training discriminative classifiers. In summary, despite the efforts involved in HC the general performance achieved for this task is still far lower than the one achieved in many other document classification tasks [19].

In this paper we propose a novel technique called Stratified Discriminant Analysis (sDA) that deals with the previously described drawback of scarcity of training examples in HC. Our model is focused on feature extraction from the text content of web documents. Here, rather than using the direct word distributions over documents and categories, and then train a set of classifiers, we advocate an initial dimensionality reduction of the data, based on Linear Discriminant Analysis (LDA) performed in a novel stratified way.

Flat LDA has been used for a while in text classification tasks [27], but as far as we know, its potential in $\mathrm{HC}$ has not yet been explored. In our sDA model, during the training phase, we perform LDA over the set of node categories in each level of the hierarchy, producing a set of projection matrices. Then, when training a node, we use the corresponding projection matrix to transform the training examples depending in which level the node is present. During testing we perform a similar procedure of feature extraction. Before a new web document 
is classified by a node, it is transformed using the transformation matrix of the corresponding level in the hierarchy. The sDA model is intended to help reduce the dimensionality of the data by keeping the most discriminant features, which could be seen as the "core" information of each category that distinguishes and groups the category from other categories on the same level of the hierarchy. This method could improve category representation, especially for categories with few training examples, which is a common case in most of the categories of a hierarchy.

We test our sDA model on the data and taxonomy underlying the K\&T dataset, extracted from the Kids\&Teens branch of the DMOZ directory. The Kids\&Teens branch embraces a diversity of topics and presents similar distributions on categories and documents as the complete DMOZ directory, which makes the K\&T dataset a suitable collection for experimentation. In order to have a better overview of the performance of SDA, we present comparisons with two models: a flat full-term classification model (FFT) and a hierarchical fullterm classification model (HFT). As indicated, both models use the whole set of terms and its direct distributions for building the classifiers. The use of direct word distributions for representing documents has proved to have good performance for HC tasks [1][4][19].

The first contribution of our work is the evidence of the feasibility of implementing LDA in a stratified way (sDA) using a decomposition per level, allowing the extraction of reduced meaningful features, which can be used along the hierarchy. The second contribution is the evidence that features extracted by the sDA technique behave very well for representing categories with few training examples in a $\mathrm{HC}$ task, and contribute to more robust classification models in those categories. Our findings open new opportunities for feature extraction and $\mathrm{HC}$ in general.

The rest of the paper is organized as follows. In section 2 we recall the related work on hierarchical classification. In section 3 we briefly describe the theory behind discriminant analysis. In section 4 we present the complete model for sDA for hierarchical classification of a web document using its textual content. Section 5 illustrates the dataset and setup used in our experiments and discusses the empirical performance of our method in comparison with the baselines. Section 6 is devoted to conclusions and ongoing research.

\section{Related Research}

$\mathrm{HC}$ has been studied in the research community for several years, and there are interesting works devoted to this task. A good survey of the work in this area is presented by Silla and Freitas in [26].

As pointed out before there are two general approaches when studying the $\mathrm{HC}$ problem [26][29], namely the flat approach and the HC approach. In the flat approach, one tries to directly categorize documents without making use of the category hierarchy. Despite the fact that a flat classifier could work for HC 
[4][20], the task of building and testing flat classifiers for large hierarchies tends to be expensive [19].

The HC classification model has been designed using several approaches. In [21], McCallum et al. proposed a hierarchical classification approach using a shrinkage method, in which smoothed parameter estimation of a data-sparse child node is used with its parent node in order to obtain robust parameter estimates. In [6], the authors presented a method based on the Fisher discriminant to rank terms and then perform feature selection by choosing only a given set of the top most discriminant words and applying this method over one part of the Yahoo! hierarchy. In [7] and [8], Dumais and Chen proposed a classifier based on SVM on the top-two levels of the LookSmart directory, called hierarchical SVM (hSVM). In [3], Cai and Hofmann proposed a hSVM based on discriminant functions that are structured in a way that mirrors the category hierarchy, obtained a superior performance than a flat SVM. Another hSVM was used on a very large-scale hierarchy in [19] over the Yahoo! directory, using a selection of local features and direct word distributions, where the hSVM uses a binary SVM classifier per node in the hierarchy, following a Pachinko-model [17][22]. Despite the relative good results obtained with the hSVM, the authors pointed out the difficulties in applying text categorization algorithms to web hierarchies, mostly because of the data scarcity problem in the rare categories of taxonomies, where most categories, especially at lower levels of the hierarchy, only have a few examples for training. Xue et al. [29] proposed a two-stage approach for HC. In a first stage, a set of category candidates are estimated for each test document. Then in a second stage, a classification model is trained using only the subset of candidate categories. This approach reduces error propagation by considering fewer categories and improves the performance of a baseline hSVM. Nevertheless, it is necessary to build a new classifier for each test example, and even if this is done in a reduced set of categories the resulting computation cost is very high. Finally Bennett and Nguyen in [1] presented a model based on hSVM but extending it to include a refinement method in order to reduce the effects of error propagation and complex decision surfaces in nodes at the top of the hierarchy. They first integrate the predictive behavior on the training data to change the training distribution in lower nodes; and they integrated a refined expert, which includes a rough class label guess in the lower-level of the hierarchy and propagates this information further up in the hierarchy. The authors report improvements compared to the results of a baseline hSVM using direct word features.

None of the above works exploit the structure of the category hierarchy for the task of feature extraction, which we propose in this paper. Rather than using the whole set of word features with their direct distributions over documents/nodes and then train the model, our approach advocates an initial dimensionality reduction of the data, based on Linear Discriminant Analysis (LDA) performed in a stratified way ( $\mathrm{sDA}$ ), with the purpose of combining all the word features into a new reduced set of attributes. The idea behind this is that sDA would produce a richer set of attributes to represent, identify and group categories than 
direct word distributions at the different levels of the hierarchy. These extracted core features might help especially in categories where limited training data are available, which is a very common case for categories inside a hierarchy, and where it is difficult to build good classification models.

In flat text classification LDA has been popular over the years as a classification/dimensionality reduction technique [14]. LDA uses the class information to project the training/testing data into a new space where the ratio of betweenclass-variance to within-class-variance is maximized in order to obtain adequate class separability. Torkkola [27] was one of the first authors to use LDA for text classification purposes. Since then, LDA in text classification has been used following several approaches: using an initial decomposition like Latent Semantic Indexing (LSI) or QR decomposition (QR) and then improved by the LDA model (LSI+LDA and QR+LDA) [14][24], Generalized Discriminant Analysis (GDA) [13][18] and Regularized Discriminant Analysis (RDA) [31]. Cai et al. [2] have proposed a new implementation for LDA, called Spectral Regression Discriminant Analysis, which is able to be linearly scalable for large datasets.

In this paper we focus solely on the effect of extracting discriminative features for the classes in the different levels of the hierarchy using sDA. We expect in this way to better group and identify the individual categories along the different levels of the hierarchy, especially the ones with very few labeled documents, reducing the effects of scarcity. For the classification itself, we rely on a topdown approach similar to a traditional hSVM. In this way our novel sDA model is complementary to other $\mathrm{HC}$ approaches described above. In future implementations we can combine the proposed feature extraction method in different classification models like the two-stage approach of [29] or the refined experts of [1], among others, in order to tackle other issues of HC like error propagation and complex decision surfaces at the top levels. To the best of our knowledge feature extraction based on the proposed sDA has not been researched in a HC task.

\section{Linear Discriminant Analysis}

$\mathrm{LDA}^{1}$ is usually defined as a transformation of data points, where the transformed data points in the new space present a good separation between classes and good proximity within each class. In this section we give a brief explanation of the main idea behind LDA.

Let $\mathbf{F}=\left\{\left(\mathbf{x}_{1}, \mathbf{y}_{1}\right),\left(\mathbf{x}_{2}, \mathbf{y}_{2}\right), \ldots,\left(\mathbf{x}_{m}, \mathbf{y}_{m}\right)\right\}$ be a set of $m$ examples with their corresponding classes, with $\mathbf{X}=\left\{\mathbf{x}_{1}, \mathbf{x}_{2}, \ldots, \mathbf{x}_{m}\right\}$, where $\mathbf{x}_{i} \in \mathbb{R}^{p}$ is the $i$-th example, represented by a $p$ dimensional row vector; and $\mathbf{Y}=\left\{\mathbf{y}_{1}, \mathbf{y}_{2}, \ldots, \mathbf{y}_{m}\right\} \subset$ $\mathbf{C}$, where $\mathbf{y}_{i} \subset\{1, \ldots, c\}$ is the set of labels for example $\mathbf{x}_{i}$ and $c=|\mathbf{C}|$. Then the $m$ documents, with $p$ features, are grouped in $c$ classes.

The goal of LDA is to find a linear transformation $\mathbf{A} \in \mathbb{R}^{p \times l}$ that projects each example vector $\mathbf{x}_{i}$ to $\mathbf{z}_{i}=\mathbf{x}_{i} \mathbf{A}$, where $\mathbf{z}$ is the projected example in a $l$

\footnotetext{
${ }^{1}$ Not to be confused with Latent Dirichlet Allocation, a topic distribution model
} 
dimensional space $(l \ll p)$, and where the class separability is intended to be maximized. In order to do so, LDA maximizes the following equation

$$
\mathbf{a}^{*}=\underset{\mathbf{a}}{\arg \max } \frac{\mathbf{a}^{T} \mathbf{B a}}{\mathbf{a}^{T} \mathbf{W a}}
$$

where $\mathbf{a}$ is a projection vector inside the matrix $\mathbf{A}$, we then look for a set of $l$ projection vectors. In the previous equation we define the between $(\mathbf{B})$ and within-group (W) covariance matrices as

$$
\begin{gathered}
\mathbf{B}=\sum_{k=1}^{c} m_{k}\left(\mu^{k}-\mu\right)\left(\mu^{k}-\mu\right)^{T} \\
\mathbf{W}=\sum_{k=1}^{c}\left(\sum_{i=1}^{m_{k}}\left(\mathbf{x}_{i}^{k}-\mu^{k}\right)\left(\mathbf{x}_{i}^{k}-\mu^{k}\right)^{T}\right)
\end{gathered}
$$

where $\mu$ is the total example mean vector, $m_{k}$ is the number of examples in the $k$-th class, $\mu^{k}$ is the average example vector of the $k$-th, and $\mathbf{x}_{i}^{k}$ is the $i$-th example in the $k$-th class. Defining $\mathbf{T}=\sum_{i=1}^{m}\left(\mathbf{x}_{i}-\mu\right)\left(\mathbf{x}_{i}-\mu\right)^{T}$ as the total covariance matrix we have $\mathbf{T}=\mathbf{B}+\mathbf{W}$. Then, equation 1 , using a complete set of $l$ projection vectors, can be expressed as

$$
\mathbf{A}^{*}=\underset{\mathbf{A}}{\arg \max } \frac{\operatorname{tr}\left(\mathbf{A}^{T} \mathbf{B} \mathbf{A}\right)}{\operatorname{tr}\left(\mathbf{A}^{T} \mathbf{T A}\right)}
$$

with $\mathbf{A}=\left[\mathbf{a}_{1}, \ldots, \mathbf{a}_{l}\right]$ and where $t r$ denotes the matrix trace. The optimization problem in (4) is equivalent to finding the $l$ eigenvectors a of the following generalized eigen-problem associated with maximum eigenvalues

$$
\mathbf{B a}=\lambda \mathbf{T a}
$$

Since the rank of $\mathbf{B}$ is bounded by $c-1$, there are at most $c-1$ eigenvectors corresponding to the non-zero eigenvalues. With the purpose of avoiding the singularity problem of matrix $\mathbf{T}$ (a singular matrix is not invertible), which could occur when the number of features is larger than the number of documents, and to cope with large datasets, in our sDA model we employ the LDA version implemented as the Spectral Regression Discriminant Analysis by Cai et al. [2].

\section{Stratified discriminant analysis}

In order to apply the discriminant analysis for the problem of $\mathrm{HC}$ of web documents, we follow a tiered approach and that is why we call our method Stratified Discriminant Analysis (sDA). Since in the actual classification we use a top-down approach for training/testing, each node is trained using examples of its descendants and we could say this step is performed and grouped per level. Hence, with the sDA feature extraction technique we intend to first better group and 
distinguish the categories at the different levels of the hierarchy by compressing the documents content in less but very meaningful features.

Given a hierarchical structure $\mathfrak{H}$, defined as a tree for this work, and a set $\mathbf{F}=\left\{\left(\mathbf{x}_{1}, \mathbf{y}_{1}\right), \ldots,\left(\mathbf{x}_{m}, \mathbf{y}_{m}\right)\right\}$ of web documents, with $\left.\mathbf{X}=\left\{\mathbf{x}_{1}, \mathbf{x}_{2}, \ldots, \mathbf{x}_{m}\right)\right\}$, where $\mathbf{x}_{i} \in \mathbb{R}^{p}$ is the $i$-th document, represented by a $p$ dimensional row vector, and $\mathbf{Y}=\left\{\mathbf{y}_{1}, \mathbf{y}_{2}, \ldots, \mathbf{y}_{m}\right\} \subset \mathbf{C}$, where $\mathbf{y}_{i} \subset\{1, \ldots, c\}$ is the set of labels for the example $\mathbf{x}_{i}$, with $c=|\mathbf{C}|$. $\mathbf{C}$ is the set of categories in $\mathfrak{H}$.

The general sDA training process is as follows. Considering that $\mathfrak{H}$ is a tree, it is composed by a number (depth) $h$ of levels. In each level of $\mathfrak{H}$ there exists a number $c_{i}$ of categories $i=1, \ldots, h$. Given a level $i$ in the hierarchy, we group the descendant categories (if the node is not a leaf) into the superior nodes of that level, in such a way that each node in the hierarchy belonging to that level $i$ is considered together with all the examples that belong to such node or its descendants. Once the examples are grouped per category at level $i$, we then apply a discriminant analysis to compute the transformation matrix $\mathbf{A}_{i}$, which has at most $l_{i}=c_{i}-1$ column dimensions. This is illustrated in figure $1 \mathrm{a}$. In this figure, $h=3$ and there are three transformation matrices computed $\mathbf{A}_{1}, \mathbf{A}_{2}, \mathbf{A}_{3}$, with column dimensions of 3,7 and 7 , respectively.

Once we have the transformation matrices, we then transform the training examples in the level $i$ using the corresponding matrix $\mathbf{A}_{i}$, obtaining a set $\mathbf{Z}_{i}$ of transformed examples, with column dimension $l_{i}$, grouped in the categories of level $i$. The last step is then to train a binary classifier in every node of level $i$. For training, we take the examples belonging to a given node (and its descendants) as the positive class and the examples belonging to its siblings (and their descendants) as the negative class, as specified in the siblings policy in [10]. This is illustrated in figure 1b. The process of grouping, applying discriminant analysis, transform examples and train classifiers, is repeated in each level $i$ of $\mathfrak{H}$, with $i=1, \ldots, h$.

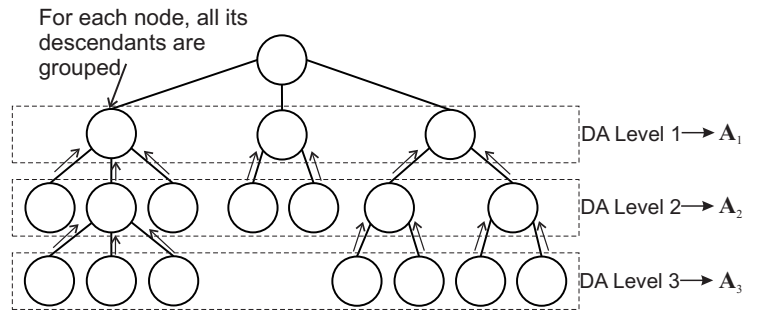

(a)

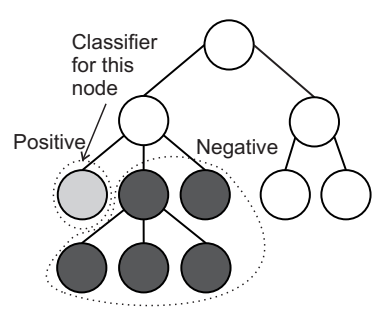

(b)

Fig. 1. Computation of discriminant analysis per level of the hierarchy (a), grouping the descendant examples into each parent category. Training a classifier for a given node of the hierarchy (b), the light grey nodes indicate the positive category and the dark grey nodes indicate the negative category. 
When assigning categories to a new, unseen, document during the testing phase, the sDA model first computes the desired transformation of the example in all the levels of the hierarchy, later a top-down approach using a Pachinkomodel [17] [22] is employed: first classifying the document at the uppermost level and then for each binary classifier that "fires" (predicts positive), classifying the document at the next lower level, and changing the representation of the document by using a new projection according to the level. Since categories are not completely disjoint, documents can belong to multiple children nodes, having a natural multi-label assignment. In our model we work with mandatory leaf-node predictions, i.e. a complete path from the root to a leaf node must be predicted. In cases where none of the children classifier assigns a category, our model assigns the most likely category according to the probabilities estimated by the classifiers, assuring a full path classification. If there are labeled documents in a non-leaf node, we insert for such non-leaf node a new child node, and all of these labeled documents on the non-leaf node will be included in the new child node. In this way, all documents are placed in leaf-node categories. The leaf mandatory prediction was imposed since in the K\&T dataset, most of the nodes are leafs. However, such restriction could be relaxed for other datasets or applications; stopping the classification of the new document in a given level, when the probability of assigning a lower-level category is less than a specified threshold.

Using the notation by Silla and Freitas [26], our sDA method could be defined as the 4-tuple $<\mathrm{T}$, MLP, MLNP, S $>$, which uses a tree (T) hierarchical structure, allows multiple labels paths (MLP), requires mandatory leaf-node prediction (MLNP), and uses siblings $(\mathrm{S})$ to define the negative examples during training. Further extensions could be easily inserted into the general model.

\section{$5 \quad$ Experimental analysis}

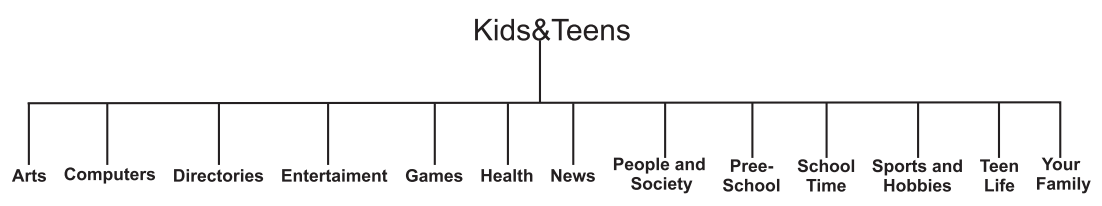

Fig. 2. First level of the hierarchy of the Kids\&Teens dataset.

\subsection{Dataset}

In order to test the validity of our sDA model, we perform tests on a dataset (K\&T dataset) obtained from the Kids\&Teens sub-category of the DMOZ directory $^{2}$ from the Open Directory Project (ODP). The hierarchy in the K\&T

\footnotetext{
2 http://www.dmoz.org/
} 
dataset, as in the whole DMOZ directory [29], is a tree, where the longest path (depth of the tree) is 10, considering the Kids\&Teens category as level 0, from where all the rest of the categories descend. In order to avoid a large number of non standard character encodings, we decided to eliminate the sub-category Kids\&Teens/International. The Kids\&Teens hierarchy up to level 1 is shown in Figure 2. It is possible to observe that this hierarchy embraces a large diversity of topics.

In order to extract the content from the web pages we took the title and description of each site from the rdf files provided by the DMOZ directory ${ }^{3}$ and then, using the links from the same rdf files, we crawled each site up to level two of the internal links in January 2012. We extracted only the textual content from the web documents, ignoring the HTML tags. As a result, we downloaded a total of 28004 web documents to form the K\&T dataset. We considered only the first 40000 characters from each document and we filtered out the stop words. For the sake of statistical significance, we wanted to perform a 5 -fold cross validation over the dataset, for that reason we eliminate categories with less than 5 documents. Finally, we filtered out again terms that appear in less than 10 documents, which is a good approach to pre-select informative words [30] and to avoid extra noise. In this way we keep a total of 20639 web documents, containing 43707 unique terms, with 2704 categories in the hierarchy, but with the documents allocated in the 2113 leaf categories. We transformed such documents into vectors using a tf-idf schema and normalized each vector to 1 . This normalized representation has shown good results for text categorization using SVM [16].

Several documents in the K\&T dataset have multiple labels. Nevertheless, as has been noticed as well for the complete DMOZ directory [23], most of the documents in the K\&T dataset are labeled with only one category. There are 20459 documents with 1 label, 169 with 2 labels and only 11 with 3 labels. On average, each document has 1.009 labels. In this case, the almost absence of multi-label documents indicates that no threshold tuning along with classifier training would be required, as suggested by [19].

From figures $3 \mathrm{a}$ and $3 \mathrm{~b}$ it is possible to observe that both categories and documents have peak distributions over levels in the K\&T dataset. That is, there are more categories and documents in the middle than at the upper and lower levels of the hierarchy. In figure $3 \mathrm{c}$ we see the relation between the number of documents and number of categories. From this graph we observe that over $40 \%$ of the categories contain 6 documents or less, and that over $60 \%$ of categories contain less than 10 documents. Similar distributions and features have been observed in the complete DMOZ directory [29]. In this sense, the K\&T dataset could be considered a good sample of the whole directory.

\subsection{Models}

As baselines for comparison with our sDA method we use two models constructed with the whole set of word features and its direct distribution (using the tf-idf

\footnotetext{
3 Available at: http://www.dmoz.org/rdf.html
} 
(a)

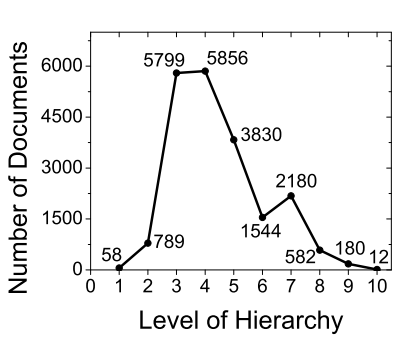

(b)

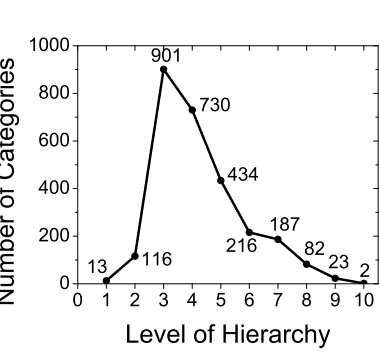

(c)

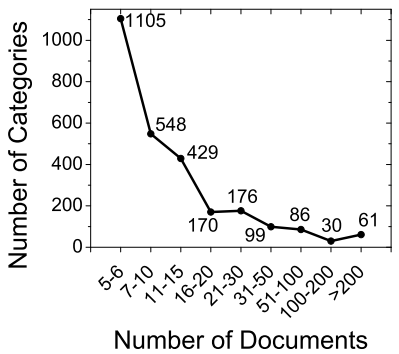

Fig. 3. Category (a) and document (b) distributions in the K\&T dataset, and relation of number of documents and number of categories (c).

vectors): a flat full-term classification model (FFT) and a hierarchical full-term classification model (HFT). The FFT model considers all the leaf-nodes as the possible categories, then it trains a binary classifier for each of them, using as positive data the documents belonging the category and as negative data all the remaining training documents, known as the all policy in [10]. During testing, it evaluates each new document for each category and assigns the categories that the classifiers assign as positive. The HFT model is built in a similar way than the sDA, as described in section 4: it trains a binary classifier for every node, taking the examples belonging to a given node (and its descendants) as the positive class and the examples belonging to its siblings (and their descendants) as the negative class. This siblings policy has presented good results for HC [10]. During testing a top-down approach is employed: first classifying the document at the uppermost level and then for each classifier that "fires" (predicts positive), classifying the document at the next lower level. The essential difference between the HFT model and the sDA model is in the use of features, i.e. level projected features for the sDA and the whole set of words distributions (tf-idf vectors) for the HFT model.

In the three models we use a linear SVM as the binary classifier in each category node. We use the implementation of the LibLinear [11] classifier, which is especially suited for training SVM in linear time. We use the following settings for the LibLinear: complexity constant C equal to 10000 (for linear SVM on text classification a large $\mathrm{C}$ tends to work fine [14]); building of logistic models, in order to obtain probabilities rather than yes/no classifications; and a threshold of 0 (separating hyperplane) for each given binary classifier to determine whether a test document belongs or not to the category. We use the wrapper for LibLinear from the Weka package [12], and the Java LibLinear library ${ }^{4}$.

All the models were implemented in Java. As mentioned before, we use the LDA implementation of the Spectral Regression Discriminant Analysis from [2]

\footnotetext{
4 Available at: http://www.bwaldvogel.de/liblinear-java/
} 
provided by the authors ${ }^{5}$. We conducted the experiments using a desktop PC with a $3.4 \mathrm{Ghz}$ Intel Core i7 processor and with $16 \mathrm{~Gb}$ in RAM.

\subsection{Performance measures}

To compare the performance of the models, we decided to use the standard label based macro precision, recall and $F 1$, which are well understood for text classification in general. These measures are recommended in [28] and [26] for multilabel HC. We use here Precision $=\frac{1}{c} \sum_{i=1}^{c} \frac{T P_{i}}{\left(T P_{i}+F P_{i}\right)}$, Recall $=\frac{1}{c} \sum_{i=1}^{c} \frac{T P_{i}}{\left(T P_{i}+F N_{i}\right)}$ and $F 1=2 \frac{\text { Precision } \cdot \text { Recall }}{\text { Precision }+ \text { Recall }}$, computed for each category $i: i=1, \ldots, c$, and which are then macro averaged. We focus on macro averaging of the performance measures because it estimates how well a model is able to perform along categories, disregarding their size [1] by weighting the performance for each class equally. In this way macro measures avoid to bias the performance towards very dense categories, neglecting or ignoring the scarce ones. Since scarce categories are very frequent in $\mathrm{HC}$ (see figure 3c) macro performance is considered as a good statistic to evaluate $\mathrm{HC}$ [1][15].

\begin{tabular}{|c|c|c|c|c|c|c|c|c|c|c|c|c|c|c|}
\hline \multicolumn{4}{|c|}{ FFT } & \multicolumn{5}{|c|}{ HFT } & \multicolumn{4}{c|}{ sDA } \\
\hline Prec & Rec & F1 & Tr & Ts & Prec & Rec & F1 & Tr & Ts & Prec & Rec & F1 & Tr & Ts \\
\hline 0.6008 & 0.6253 & 0.5916 & 8150 & 574 & 0.6085 & 0.6262 & 0.5945 & $\mathbf{6 2 5}$ & $\mathbf{4 6}$ & $\mathbf{0 . 6 1 6 7}$ & $\mathbf{0 . 6 3 7 0}$ & $\mathbf{0 . 6 0 3 5}$ & 1762 & 189 \\
\hline
\end{tabular}

Table 1. Performance of the models in terms of precision (Prec), recall (Rec), F1, training (Tr) and testing (Ts) time. The times are expressed in seconds.

\subsection{Results}

The comparative results of the FFT, HFT and sDA models for the experiments with a 5 -fold cross validation on the K\&T hierarchy are summarized in table 1. The performance in terms of recall, precision and F1 measures is computed using the true most specific categories and all their ancestor categories, and the most specific predicted categories and all their ancestors for each web document. The quantities in the table are averages over the 5 folds. We have performed onesided Wilcoxon signed rank tests for each performance measure across the folds, testing the hypothesis of $F(x)>G(y)$, i.e. if the values of one method tend to be better than the ones of other method. All the differences for the sDA model in comparison with the FFT and HFT models are significant with $p<0.05$. The differences between the FFT and the HFT models are only significant for precision with $p<0.05$ but are not significant for recall and F1. That means that the FFT and the HFT models perform similarly for such measures. Nevertheless, the the HFT model performs much better than the FFT model regarding the time complexity. The HFT model performs one order of magnitude faster than

\footnotetext{
$\overline{5}$ Available at: http://www.zjucadcg.cn/dengcai/SR/index.html
} 
the FFT model, both for training and testing. In this sense, the benefit of using a hierarchical version of SVM over a flat version is evident. sDA outperforms both state-of-the-art methods in performance but is slower than the HFT model. The sDA model is more expensive because its training time includes the computation of the transformation matrices and the transformation of examples using such matrices in the different levels of the hierarchy. During testing the sDA method needs to perform the transformation of each testing example along the different levels of the hierarchy using the computed matrices. Nevertheless, we observe that the times are still feasible and less than the ones of the FFT model.

In figure 4 the results of the FFT, HFT and sDA models are split per level of the hierarchy according to the average performance of the 5 folds. The performance per level is computed using the true and predicted categories from the root to the given level. In this sense, it is a cumulative performance and the results of level 10 correspond to the ones shown in table 1, which measures the performance in the whole hierarchy.

(a)

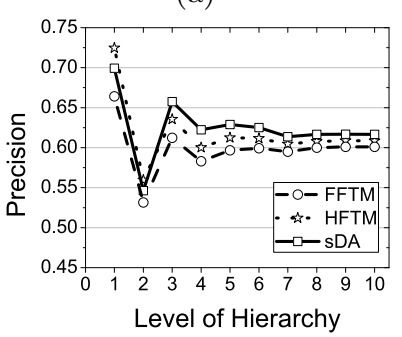

(b)

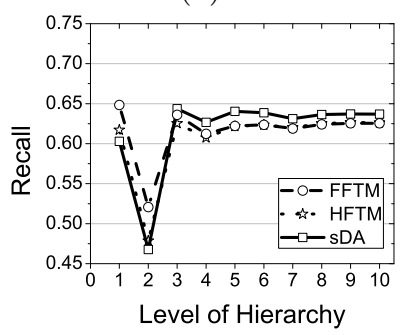

(c)

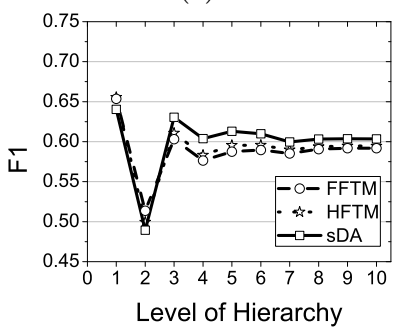

Fig. 4. Performance of the FFT, HFT and sDA models in terms of precision (a), recall (b) and F1 (c) split per level of the hierarchy.

The best performance for all the methods is reached at level 1 , where there are few categories and most of them contain a large number of examples (given that the examples are collected in the parent nodes). In this level, FFT and HFT models perform slightly better than sDA for F1 (figure 4c), given that with categories at the top level, the effect of a mixture of topics is more evident, and the use of larger vocabularies could better discriminate among them. Similarly at level 2, the FFT and HFT models perform slightly better than sDA because the number of categories is still low and there are more dense categories with overlapping content. At level 2 there is a dip, which could be attributable to the presence of very broad categories like teen_life/online_communities, teen_life/issues, pre_school/people, pre_school/animals, etc. whose content could be assigned to other more specific or descriptive categories.

In level 3 we have the maximum number of categories, and given the general large amount of categories with few training examples (see figure 3c), the sDA model shows its best performance here, meaning that at this level there are 
many (leaf) categories with few training examples. At levels 4, 5 and 6 we find the majority of the categories. In those levels we still have a large number of scarce categories but less than at level 3. For that reason, the performance of the sDA is better than the FFT and the HFT models but the difference is less in comparison with level 3. As the general number of categories decreases, from level 7 , so does the number of scarce categories and the differences in performance between methods does as well, but in general sDA still keeps its better performance than FFT and HFT models.

The effect of scarcity of training examples in categories (i.e. the number of positive labeled documents) on the performance of the methods is shown in figure 5, which plots the precision, recall and F1 depending on the number of training documents in the category. It is clear that for categories with few training examples (between 4 and 6, which represent over $40 \%$ of the total number of categories) the sDA model outperforms the FFT model and the HFT model. Especially with only 4 documents, sDA reaches its best performance. This means that the transformation involved in sDA is very well suited to build reduced representations of categories with only a few training examples. As the number of available training documents increases so does the performance of all the models. It is clear as well, that the FFT and HFT models perform better when they use more training documents, since they are able to better generalize with the use of large word distribution features. Nevertheless, on those cases the performance of sDA is still competitive.

(a)

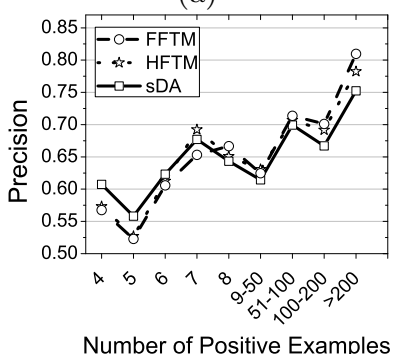

(b)

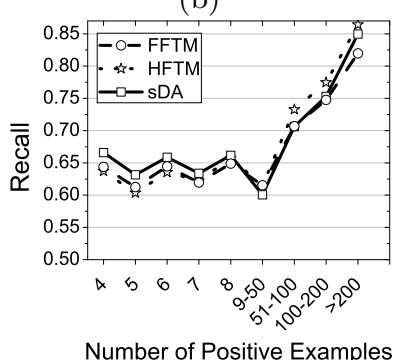

(c)

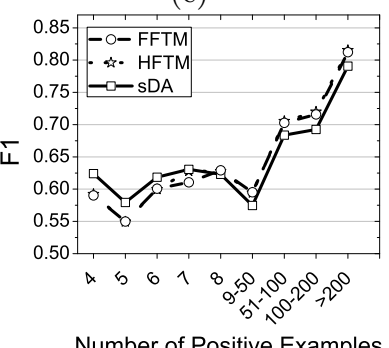

Fig. 5. Performance of the FFT, HFT and sDA models in terms of precision (a), recall (b) and F1 (c) vs. the number of positive examples in the training set.

\section{Conclusions and future work}

Our research adds value to a growing body of work exploring how hierarchies structures can be used to improve the efficiency and efficacy of classification of web documents based on their contents. In this article we have presented a method that focuses on a novel feature extraction technique named Stratified 
Discriminant Analysis (sDA) that exploits the knowledge resided in the hierarchy for extracting compressed features from the word features of the documents.

Our conclusions are that sDA offers advantages over a flat classification model and a hierarchical classification model which use direct word distributions as features. The sDA model reaches a better performance for precision, recall and F1 and keeps the computation time feasible. More specifically, we observed that the sDA model helps separating the different categories in each level of the hierarchy. This model especially improves the grouping and representation of web pages for categories where the number of training examples is scarce. Categories with few training examples are indeed very common in $\mathrm{HC}$ and are normally neglected or ignored in benefit of more dense categories. The results presented are truly encouraging and have shown that sDA has the potential to be exploited for the tasks of classifying documents into a hierarchy.

There are many directions to further explore with the sDA model. We are firstly interested into extending the model for larger hierarchies, since the implementation of LDA used in the Spectral Regression model scales well for large datasets, this is feasible. Moreover, with larger hierarchies, the problem of scarcity will become more evident [19], and sDA could be useful. As pointed out, we are interested as well, into combining word features with the features extracted by the sDA model. This could be done by performing sDA over scarce categories and use word features in dense categories; or by linearly combining both type of features into one single model. Another path is the use of sDA in combination with methods that avoid error propagation from top levels, such as the afore mentioned refined experts by [1], the two-stage approach by [29], or the use of different ways of selecting the negative examples during training.

\section{Acknowledgments}

This research was supported partially by the CONACYT postdoctoral grant I0010-2010-01/150813, and by the EU FP7-231507 PuppyIR project.

\section{References}

1. P. N. Bennett and N. Nguyen. Refined experts: improving classification in large taxonomies. In Proc. 32nd ACM SIGIR, pages 11-18. ACM Press, 2009.

2. D. Cai, X. He, and J. Han. Srda: An efficient algorithm for large-scale discriminant analysis. IEEE Transactions on Knowledge and Data Engineering, 20(1):1-12, 2008.

3. L. Cai and T. Hofmann. Hierarchical document categorization with support vector machines. In Proc. 13th ACM CKIM, pages 78-87. ACM Press, 2004.

4. M. Ceci and D. Malerba. Classifying web documents in a hierarchy of categories: a comprehensive study. Journal of Intelligent Information Systems, 28(1):37-78, 2007.

5. N. Cesa-Bianchi, C. Gentile, and L. Zaniboni. Incremental algorithms for hierarchical classification. Journal Machine Learning Reasearch, 7:31-54, 2006. 
6. S. Chakrabarti, B. E. Dom, R. Agrawal, and P. Raghavan. Scalable feature selection, classification and signature generation for organizing large text databases into hierarchical topic taxonomies. Journal of Very Large Data Bases, 7(3):163-178, 1998.

7. H. Chen and S. Dumais. Bringing order to the web: automatically categorizing search results. In Proc. SIGCHI conference, pages 145-152. ACM Press, 2000.

8. S. Dumais and H. Chen. Hierarchical classification of web content. In Proc. 23rd ACM SIGIR, pages 256-263. ACM Press, 2000.

9. S. Dumais, E. Cutrell, and H. Chen. Optimizing search by showing results in context. In Proc. SIGCHI conference, pages 277-284. ACM Press, 2001.

10. T. Fagni and F. Sebastiani. Selecting negative examples for hierarchical text classification: an experimental comparison. Journal of the American Society for Information Science, 61(11):2256-2265, 2010.

11. R. E. Fan, K. W. Chang, C. J. Hsieh, X. R. Wang, and C. J. Lin. Liblinear: a library for large linear classification. Journal of Machine Learning Research, 9:1871-1874, 2008.

12. M. Hall, E. Frank, G. Holmes, B. Pfahringer, P. Reutemann, and I. H. Witten. The WEKA data mining software: an update. ACM SIGKDD Explorations Newsletter, 11(1):10-18, 2009.

13. P. Howland and H. Park. Generalizing discriminant analysis using the generalized singular value decomposition. IEEE Transactions on Pattern Analysis and Machine Intelligence, 26(8):995-1006, 2004.

14. H. Kim, P. Howland, and H. Park. Dimension reduction in text classification with support vector machines. Journal of Machine Learning Research, 6:37-53, 2005.

15. A. Kosmopoulos, E. Gaussier, G. Paliouras and S. Aseervatham. The ECIR 2010 large scale hierarchical classification workshop. 2010

16. M. Lan, C. L. Tan, H.-B., Low and S. Yuan. A comprehensive comparative study on term weighting schemes for text categorization with support vector machines. In Proc. 14th $W W W$, pages 1032-1033. 2005.

17. W. Li and A. McCallum. Pachinko allocation: Dag-structured mixture models of topic correlations. In Proc. 23rd ICML, pages 577-584. ACM Press, 2006.

18. T. Li, S. Zhu and M. Ogihara. Text categorization via generalized discriminant analysis. Information Processing and Management, 44(5):1684-1697, 2008.

19. T.-Y. Liu, Y. Yang, H. Wan, H.-J. Zeng, Z. Chen, and W.-Y. Ma. Support vector machines classification with a very large-scale taxonomy. ACM SIGKDD Explorations Newsletter, 7(1):36-43, 2005.

20. H. Malik. Improving hierarchical SVMS by hierarchy flattening and lazy classification. In Proc. Large-Scale Hierarchical Classification Workshop of ECIR, 2010.

21. A. McCallum, R. Rosenfeld, T. M. Mitchell, and A. Y. Ng. Improving text classification by shrinkage in a hierarchy of classes. In Proc. 15th ICML, pages 359-367. Morgan Kaufmann Publishers Inc., 1998.

22. D. Mimno, W. Li, and A. McCallum. Mixtures of hierarchical topics with Pachinko allocation. In Proc. 24th ICML, pages 633-640. ACM Press, 2007.

23. G. Paliouras, E. Gaussier, A. Kosmopoulos, I. Androutsopoulos, T. Artieres and P. Gallinari. Joint ECML/PKDD PASCAL workshop on large-scale hierarchical classification. 2011

24. C. H. Park and M. Lee. On applying linear discriminant analysis for multi-labeled problems. Pattern Recognition Letters, 29(7):878-887, 2008.

25. X. Qi and B. D. Davidson. Web page classification: features and algorithms. ACM Computing Surveys, 41(2):1-31, 2009. 
26. C. Silla and A. Freitas. A survey of hierarchical classification across different application domains. Data Mining and Knowledge Discovery, 22(1):31-72, 2011.

27. K. Torkkola. Linear discriminant analysis in document classification. In Proc. IEEE ICDM Workshop on Text Mining. IEEE, 2001.

28. G. Tsoumakas, I. Katakis and I. Vlahavas. Mining multi-label data. Data Mining and Knowledge Discovery Handbook, chapter 34, pages 667-685, 2010

29. G. R. Xue, D. Xing, Q. Yang and Y. Yu. Deep classification in large-scale text hierarchies. In Proc. 31st ACM SIGIR, pages 619-626. ACM Press, 2008.

30. Y. Yang and J. O. Pedersen. A comparative study on feature selection in text categorization. In Proc. 14th ICML, pages 412-420. Morgan Kaufmann Publishers Inc., 1997.

31. J. Yen and T. Wang. Regularized discriminant analysis for high dimensional, low sample size data. In Proc. 12th ACM SIGKDD, pages 454-463. ACM Press, 2006. 